
factors and association with daily activities among pregnant women in urban Blantyre, Malawi

\section{Steven D. Manyozo ${ }^{1,4}$, Tarimo Nesto ${ }^{2}$, Phylos Bonongwe $^{3}$, Adamson S. Muula ${ }^{1,4}$}

1. Department of Public Health, College of Medicine, University of Malawi

2. Department of Physiotherapy, College of Medicine, University of Malawi

3. Department of Obstetrics and Gynecology, Queen Elizabeth Central Hospital, Malawi Ministry of Health

4. Africa Center of Excellence in Public Health \& Herbal Medicine, College of Medicine, University of Malawi

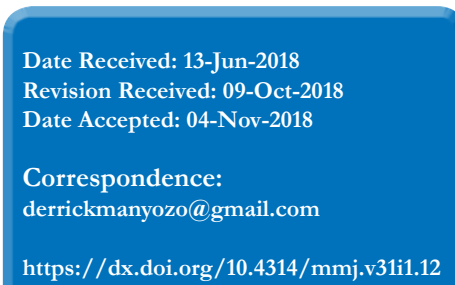

Introduction

\title{
Abstract
}

Low back pain (LBP) is a significant musculoskeletal problem during pregnancy with potential to negatively affect the woman's quality of life. Data on LBP among pregnant women in Malawi is almost non-existent. We investigated the prevalence and risk factors of LBP and its association with functional activities in pregnant women in Malawi.

Methods

We conducted a cross-sectional study in Blantyre, Malawi, from December 2017 to January 2018. Participants were drawn from low-risk antenatal clinics in selected local health facilities. Written informed consent was sourced from study participants, permission was granted at each study site and the study received ethics approval from the College of Medicine Research Ethics Committee (COMREC). Descriptive statistics were used to summarize the data. Categorical variables were summarized as frequencies and percentages. The association between occurrence of LBP and selected factors was assessed using the Chi-Square test $\left(\mathrm{X}^{2}\right)(\alpha=5 \%)$ followed by a multiple logistic regression. Odds ratios (OR) and their 95\% confidence intervals were calculated.

Results

We interviewed 404 pregnant women; the mean age of respondents was 25.83 years old (SD: \pm 5.91 ). Prevalence of LBP in pregnancy was $62 \%(n=249) ; 172(69 \%)$ of these reported LBP for the first time during the current pregnancy. Gestational age was significantly associated with presence of LBP $(P=0.03)$. LBP was associated with the women's sleep patterns, mobility, lifting techniques and sexual activities. However, a reasonable high proportion of those with LBP $(34 \%)$ did not seek care for their low back pain.

Conclusion

LBP is highly prevalent and an important clinical condition among pregnant women in Blantyre, Malawi. Given the significant effect of LBP on quality of life, health workers need to be proactive in identifying LBP and provide the appropriate management.

Keys words: Low back pain, Prevalence, Pregnancy, Activity limitations, Malawi

\section{Introduction}

Low back pain (LBP) is a common musculoskeletal problem during pregnancy with an estimated prevalence ranging from $30 \%$ to $78 \%$ in the United States of America, Europe and some parts of Africa ${ }^{1,2,3}$. One third of the population suffering from LBP report severe pain which is often associated with limitations on a woman's ability to work effectively, leading to poor quality of $\operatorname{life}^{1}$. Consequently, the woman's individual productivity in their daily routine activities is reduced ${ }^{4}$. Many of the women with LBP experience their first episode of LBP during pregnancy ${ }^{4}$. Despite the disabling effects of LBP in pregnancy, LBP is often untreated and considered normal and inevitable part of pregnancy among women ${ }^{1,4}$. The exact cause of LBP in pregnancy is poorly understood, often considered multifactorial in nature, and associated with biomechanical, vascular and hormonal changes during pregnancy 3,5 . There are varied practices in the management of pregnancy related $\mathrm{LBP}^{3}$. There is currently no consensus regarding the risk factors for LBP in pregnancy. However, pelvic trauma, young age, multiparous, chronic LBP and history of LBP in the previous pregnancy ${ }^{5,6}$ have been indicated as the most common risk factors for LBP in pregnancy ${ }^{4}$.

The health risks associated with the use of analgesics in pregnancy are well documented ${ }^{7}$. For example, Non-Steroidal Anti-Inflammatory Drugs (NSAIDs) are contraindicated in the third trimester of pregnancy and opioids are not considered safe throughout pregnancy. Therefore, management of LBP using drugs during pregnancy has not been satisfactory ${ }^{7}$. As a result, many women consider LBP as an inevitable normal discomfort to live with during pregnancy ${ }^{5,8}$. However, non-pharmacological interventions such as soft tissue manipulation, postural education, stabilization exercises, and transcutaneous electrical nerve stimulation, often provided by physiotherapists, have been found to be effective in managing LBP during pregnancy and 
has since been recommended as first line of management for the treatment of LBP in pregnancy ${ }^{5}$.

There is paucity of data on LBP in Malawi including LBP characterization and clinical course. In 2014, Tarimo and Diener ${ }^{9}$ reported that the majority of patients with chronic low back pain at two tertiary health facilities in Malawi held negative attitudes and beliefs about their LBP. However, we did not find any prevalence data on LBP in Malawi, particularly LBP in pregnant women. Nonetheless, LBP in pregnancy is an important clinical condition requiring proper attention and management to improve the woman's quality of life and individual productivity ${ }^{9}$. Understanding the prevalence and risk factors for LBP during pregnancy and its associations with activities of daily living in pregnancy is critical to informing a comprehensive antenatal care and improving the quality of clinical care offered to pregnant women. ${ }^{10}$ This study, therefore, aimed to determine the prevalence and risk factors for LBP and assess its implication on functional activities among pregnant women Malawi.

\section{Methods \\ Study design and setting}

We conducted a quantitative cross-sectional study. The study was conducted within 5 local health facilities in Blantyre urban, Malawi, namely: Queen Elizabeth Central Hospital and Zingwangwa, Limbe, Chileka and Chilomoni health centers. The list of health facilities was obtained from Blantyre District Health Office. Urban Blantyre in our setting refers to areas within the Blantyre district headquarters and those within designated town centers. ${ }^{11}$

\section{Sample size and sampling methods}

The sample size was estimated using the Cochran's formula at an estimated low back pain prevalence of $50 \%{ }^{12}, 5 \%$ precision level within a $95 \%$ confidence $(Z=1.96)$. Data were collected from December 2017 to January 2018. Using the RANDBETWEEN function in Microsoft excel 2016, a simple random sample of 5 local health facilities was selected from a list of 10 health facilities in Blantyre urban. Study participants were drawn from low risk antenatal clinics within urban Blantyre at Queen Elizabeth Central Hospital and Zingwangwa, Limbe, Chileka and Chilomoni health centers. The low risk antenatal clinics are normally managed by a general medical officer and/or clinical officer ${ }^{13}$. Using consecutive sampling, a proportional sample from each facility was obtained. Respondents were included in the study if they were aged 16 years and older, were attending antenatal clinics in Blantyre urban, able to comprehend and consent. Women with history of trauma and those with mental impairment were excluded.

\section{Ethical review and approval}

The study was reviewed and approved by the College of Medicine Research Ethics Committee (COMREC), (certificate number P.09/17/2286). Permission to conduct the study at the indicated study sites was granted by the Director of Queen Elizabeth Central Hospital and the District Health Officer for Blantyre. To ensure privacy and confidentiality, codes were used on the questionnaire instead of participants' names. An impartial witness was invited to consent for participants who could not read and write. Young women below the age of 18 were considered emancipated minors on account of their being pregnant. Research assistants were trained on the purpose of the study and all ethical requirements for the study.

\section{Data collection}

A researcher administered questionnaire was used to collect data from pregnant women who were attending the antenatal clinics and consented to participate in the study. The questionnaire used in this study was developed by our study group, we adapted and modified a validated questionnaire that was used in the United States (US) to assess prevalence of LBP among pregnant women ${ }^{4}$. Content validity was ensured by experts working in the field of LBP including two physiotherapists, two medical doctors, one obstetrician and one epidemiologist. For face validity, a pilot study was conducted on 20 pregnant women to ascertain the simplicity and clarity of questions in the questionnaire and the time it would take to be completed by the participant. The questionnaire was translated into Chichewa and collected data on (1) social demographic characteristics regarding participant's age, marital status, occupation, education, location and pre-existing conditions, (2) respondents' past experiences with LBP and LBP during the current pregnancy. For those who reported LBP in the current pregnancy, questions regarding aggravating factors were assessed. The Oswestry Low Back Pain Disability Questionnaire was used to collect data on functional status ${ }^{14}$. This is a valid and reliable tool for assessing functional limitations associated with LBP in different populations. Its internal consistency has been shown to be of acceptable standard by different authors with a Cronbach $\alpha$ value of .71 to .87 and a high test retest reliability, $r=0.83$ to $0.99^{14,15}$.

\section{Data analysis}

Data were analyzed in Stata statistical software version 14. Descriptive statistics were summarized using frequencies and percentages. The Chi square was used to test for any significant associations between categorical variables and LBP, and the significance level was set at 5\%. A logistic regression model was then fitted to assess the effects of covariates; maternal age, marital status, educational level, gravidity and gestational age on LBP. Crude and adjusted Odds ratios (OR) were obtained to quantify the probability of developing LBP during pregnancy.

\section{Results}

\section{Socio-demographic characteristics of the study participants}

Out of the 405 questionnaires that were administered in the selected antenatal clinics, one questionnaire contained missing information and was excluded. The remaining 404 questionnaires entered into a database for analysis. The mean age for the respondents was 25.83 years old (SD: \pm 5.91 ). Of the 404 respondents, $393(97 \%)$ had attended school at different levels while the remaining 3\% (11) never went to school. Of those who attended school, $230(57 \%)$ women had attended secondary education, $92(23 \%)$ women had attended primary school and $71(18 \%)$ had attended tertiary 
schools. Majority ( $\mathrm{n}=366,91 \%$ ) of the women were married living in established homes and only 9\% were not married. Among all the respondents, 154 (38\%) were visiting the antenatal clinics for their first pregnancy and the rest were coming for their review antenatal visit. Almost half of the respondents $(\mathrm{n}=179,49 \%)$, were in the third trimester and $173(43 \%)$ were in the second trimester of their pregnancy. Most of our respondents in this study did not report any comorbid conditions except $22(5.5 \%)$ women who reported living with asthma.

\section{Prevalence of low back pain among the study participants}

Out of the 404 respondents, $249(62 \%)$ pregnant women reported having LBP during the current pregnancy. Majority $(\mathrm{n}=172,69 \%)$ of the women that reported having LBP were experiencing LBP for the first time during the current pregnancy. However, one in three women $(n=123,31 \%)$ reported episodes of lower back pain prior to the current pregnancy, $59(48 \%)$ of these women with previous LBP experienced LBP during their menstrual periods and 37 (30\%) reported nonspecific low back pain prior to their pregnancy. Table 1 below shows a summary of low back pain prevalence and their 95\% confidence intervals.

Table 1: Distribution of low back pain among the respondents

\begin{tabular}{llll}
\hline & $\begin{array}{l}\text { Frequency } \\
(\mathrm{n}=404)\end{array}$ & Proportion (\%) & $\begin{array}{l}95 \% \text { confidence } \\
\text { interval }\end{array}$ \\
\hline $\begin{array}{l}\text { Women with } \\
\text { LBP }\end{array}$ & 249 & 61.63 & $57.07-66.58$ \\
$\begin{array}{l}\text { Women without } \\
\text { LBP }\end{array}$ & 155 & 38.37 & $33.42-42.93$ \\
\hline
\end{tabular}

\section{Risk factors}

Table 2 below shows the distribution of selected socio demographic factors and their subgroup prevalence of LBP in pregnancy. LBP prevalence was increasing with increasing education levels, increasing gestational age in trimesters, increasing gravidity, decreasing maternal age and the status of being married (Table 3). Women who were in their second (adjusted OR 1.83, $\mathrm{p}=.12$ ) and third trimesters (adjusted OR 2.35, $\mathrm{p}=.03$ ) were more likely to report LBP compared to those in first trimester. Those who attended formal education at secondary (adjusted OR 1.13, $\mathrm{p}=.85$ ) and tertiary (adjusted OR 1.30, $\mathrm{p}=.69$ ) education levels were more likely to report LBP compared to those with no education at all. However, only gestational age in trimesters was significantly associated with LBP and maternal age; marital status, education levels and gravidity were not significantly associated with the occurrence of low back pain in the study population when tested at $5 \%$ significant level.

\section{Impact of LBP on activities of daily living}

Low back pain poses a significant challenge among preg- nant women regarding their activity of daily living. In this study, 54 (24\%) women among those with LBP reported not being able to walk more than 100 yards due to their LBP, and $49(21 \%)$ women were not able to do the usual lifting of objects which they were able to before pregnancy.

Table 2: Characteristics of respondents by socio-demographic characteristics according to Low Back Pain status $(n=404)$

\begin{tabular}{|c|c|c|c|}
\hline \multirow[t]{2}{*}{ Factor } & Total & $\begin{array}{l}\text { With Low } \\
\text { Back Pain }\end{array}$ & $\begin{array}{l}\text { Without } \\
\text { Low Back } \\
\text { Pain }\end{array}$ \\
\hline & $\mathrm{N}(\%)$ & $\mathrm{N}(\%)$ & $\mathrm{N}(\%)$ \\
\hline \multicolumn{4}{|l|}{ Age (Years) } \\
\hline $16-20$ & $86(21 \%)$ & $56(65 \%)$ & $30(35 \%)$ \\
\hline $21-25$ & $137(34 \%)$ & $89(65 \%)$ & $48(35 \%)$ \\
\hline $26-30$ & $90(22 \%)$ & $54(60 \%)$ & $36(40 \%)$ \\
\hline $31-41$ & $91(23 \%)$ & $50(55 \%)$ & $41(45 \%)$ \\
\hline \multicolumn{4}{|l|}{ Marital Status } \\
\hline Single & $38(9.0 \%)$ & $22(58 \%)$ & $16(42 \%)$ \\
\hline Married & $366(91.0 \%)$ & $227(62 \%)$ & $139(38 \%)$ \\
\hline \multicolumn{4}{|l|}{ Educational Levels } \\
\hline None & $11(3 \%)$ & $6(55 \%)$ & $5(45 \%)$ \\
\hline Primary & $92(23 \%)$ & $53(58 \%)$ & $39(42 \%)$ \\
\hline Secondary & $230(57 \%)$ & $144(63 \%)$ & $84(37 \%)$ \\
\hline Tertiary & $71(18 \%)$ & $46(65 \%)$ & $25(35 \%)$ \\
\hline \multicolumn{4}{|l|}{ Gravidity } \\
\hline Primigravida & $154(38 \%)$ & $97(63 \%)$ & $55(37 \%)$ \\
\hline Multigravida & $250(62 \%)$ & $152(61 \%)$ & $98(39 \%)$ \\
\hline \multicolumn{4}{|l|}{ Gestational Age } \\
\hline First trimester & $32(8 \%)$ & $15(47 \%)$ & $17(53 \%)$ \\
\hline Second trimester & $173(43 \%)$ & $104(60 \%)$ & $69(40 \%)$ \\
\hline Third trimester & $199(49)$ & $132(66 \%)$ & 67 (34\%) \\
\hline
\end{tabular}

Furthermore, 53(22\%) women reported not being able to sit for more than 10 minutes and $5(2 \%)$ of them could not sit at all due to LBP. Among the women who reported LBP, 12 $(5 \%)$ reported experiencing fairly severe pain such that 17 $(7 \%)$ women became very slow at doing personal care activities to avoid causing extra pain during the activity.

Among the women with LBP, 107 (43\%) reported sleep disturbances, $34(14 \%)$ of these were only able to sleep less for than 2 hours, $25(10 \%)$ were able to sleep for an average of 4 hours and the majority reported occasional sleep disturbance as pain woke them up at night. In addition, 85 (34\%) women had a difficult sexual experience due to LBP $17(20 \%)$ of whom reported very painful sexual activities, 11 $(12 \%)$ women had severely restricted sexual life and $4(2 \%)$ nearly had no sex at all due to LBP. Other areas affected included social participation whereby $11(5 \%)$ women were restricted to their home environment, and $14(6 \%)$ women were only able to travel for treatment due to severe LBP. Of the women with LBP, $49(21 \%)$ could not stand for more 
than 10 minutes and $7(3 \%)$ could not stand at all.

\section{Management of low back pain and coping strategies}

Among all women that reported LBP in the current pregnancy, forward flexion and sitting were the most reported pain aggravating factors affecting $77(31 \%)$ and $52(21 \%)$ respectively. Resting from the pain aggravating activity, doing opposite activities to the ones causing pain and use of analgesics were the most frequent pain alleviating modalities and coping strategies among those with LBP, used by $85(34 \%), 30(12 \%)$ and $22(9 \%)$ women respectively. Other modalities including massage, stretching and doing general physical exercise were used following a pain episode. However, 85 (34\%) women did nothing to relieve their LBP (Table 4). Of all respondents, $113(28 \%)$ were coming in to the antenatal clinic for the first consultation. Of the 291 $(72 \%)$ who were coming for their review consultations, only $32(11 \%)$ had been asked specifically about any experience of LBP in their previous visits by the midwives and/or the physicians.

Table 3. Factors Associated with low back pain in pregnancy. Results from a logistic regression

\begin{tabular}{|c|c|c|}
\hline Factor & $\begin{array}{l}\text { unadjusted OR } \\
(95 \% \mathrm{Cl})\end{array}$ & $\begin{array}{l}\text { Adjusted OR }(95 \% \\
\mathrm{Cl})\end{array}$ \\
\hline \multicolumn{3}{|l|}{ Age (Years) } \\
\hline $16-20$ & $1(\mathrm{ref})^{*}$ & \\
\hline $21-25$ & $1.04(0.59-1.83)$ & $0.95(0.50-1.79)$ \\
\hline $26-30$ & $0.80(0.44-1.48)$ & $0.71(0.34-1.51)$ \\
\hline $31-41$ & $0.65(0.36-1.20)$ & $0.53(0.25-1.15)$ \\
\hline \multicolumn{3}{|l|}{ Marital Status } \\
\hline Married & $1(\mathrm{ref})^{*}$ & \\
\hline Single/separated & $0.96(0.48-1.94)$ & $0.87(0.42-1.84)$ \\
\hline \multicolumn{3}{|l|}{ Education } \\
\hline None & $1(\mathrm{ref})^{*}$ & \\
\hline Primary & $1.13(0.32-3.98)$ & $0.85(0.23-3.13)$ \\
\hline Secondary & $1.42(0.42-4.82)$ & $1.13(0.32-3.94)$ \\
\hline Tertiary & $1.53(0.42-5.53)$ & $1.30(0.35-4.83)$ \\
\hline \multicolumn{3}{|l|}{ Gravidity } \\
\hline Primigravida & $1(\mathrm{ref})^{*}$ & \\
\hline Multigravida & $0.88(0.58-1.3)$ & $1.13(0.64-2.0)$ \\
\hline \multicolumn{3}{|l|}{ Gestational age } \\
\hline First trimester & $1(\mathrm{ref})^{*}$ & \\
\hline Second trimes- & $1.7(0.80-3.63)$ & $1.83(0.85-3.95)$ \\
\hline Third trimester & $2.2(1.04-4.71)$ & $2.35(1.09-5.06)^{* *}$ \\
\hline
\end{tabular}

\section{Impact of LBP on activities of daily living}

Low back pain poses a significant challenge among preg- nant women regarding their activity of daily living. In this study, 54 (24\%) women among those with LBP reported not being able to walk more than 100 yards due to their LBP, and $49(21 \%)$ women were not able to do the usual lifting of objects which they were able to before pregnancy. Furthermore, 53(22\%) women reported not being able to sit for more than 10 minutes and $5(2 \%)$ of them could not sit at all due to LBP. Among the women who reported LBP, $12(5 \%)$ reported experiencing fairly severe pain such that $17(7 \%)$ women became very slow at doing personal care activities to avoid causing extra pain during the activity.

Among the women with LBP, 107 (43\%) reported sleep disturbances, $34(14 \%)$ of these were only able to sleep less for than 2 hours, $25(10 \%)$ were able to sleep for an average of 4 hours and the majority reported occasional sleep disturbance as pain woke them up at night. In addition, 85 (34\%) women had a difficult sexual experience due to LBP $17(20 \%)$ of whom reported very painful sexual activities, $11(12 \%)$ women had severely restricted sexual life and 4 $(2 \%)$ nearly had no sex at all due to LBP. Other areas affected included social participation whereby $11(5 \%)$ women were restricted to their home environment, and $14(6 \%)$ women were only able to travel for treatment due to severe LBP. Of the women with LBP, $49(21 \%)$ could not stand for more than 10 minutes and $7(3 \%)$ could not stand at all.

Table 4: Pain management and coping strategies for pregnant women with $\operatorname{LBP}(\mathrm{n}=249)$

\begin{tabular}{lll}
\hline Coping Strategies & Total $(\mathrm{n})$ & Percent total \\
\hline Doing opposite activity & 29 & $12 \%$ \\
Rest from an activity & 85 & $34 \%$ \\
Pain medication & 23 & $9 \%$ \\
Back massage & 4 & $2 \%$ \\
Nothing & 84 & $34 \%$ \\
Physical exercise & 13 & $5 \%$ \\
Stretching & 11 & $4 \%$
\end{tabular}

\section{Management of low back pain and coping strategies}

Among all women that reported LBP in the current pregnancy, forward flexion and sitting were the most reported pain aggravating factors affecting $77(31 \%)$ and $52(21 \%)$ respectively. Resting from the pain aggravating activity, doing opposite activities to the ones causing pain and use of analgesics were the most frequent pain alleviating modalities and coping strategies among those with LBP, used by $85(34 \%), 30(12 \%)$ and $22(9 \%)$ women respectively. Other modalities including massage, stretching and doing general physical exercise were used following a pain episode. However, 85 (34\%) women did nothing to relieve their LBP (Table 4). Of all respondents, $113(28 \%)$ were coming in to the antenatal clinic for the first consultation. Of the 291 $(72 \%)$ who were coming for their review consultations, only $32(11 \%)$ had been asked specifically about any experience of LBP in their previous visits by the midwives and/or the 
physicians.

\section{Discussion}

A pregnant woman undergoes many anatomical and physiological changes throughout the gestation period. These changes affect the musculoskeletal system causing pain and discomfort in the lower $\mathrm{back}^{8}$. Results from this study show that LBP is a common problem among pregnant women in Malawi and they agree with studies done in other countries that indicate that at least $50 \%$ of pregnant women have low back pain ${ }^{3,8}$. In our study, at least 2 in every 3 pregnant women reported back pain and this was 2 times higher compared to findings from a recent 2018 study report on pregnancy related LBP in Ethiopia ${ }^{2}$. However, our results closely agree with findings from a 2017 study conducted in Brazil where a 68\% LBP prevalence was reported ${ }^{3}$.

This study also shows that LBP can start at any point during pregnancy from first through to the third trimesters. However, women in the second (adjusted OR 1.83, $p=.12$ ) and third trimesters (adjusted OR 2.35, $p=.03$ ) have higher odds of having LBP compared to women in their first trimester. These results further agree with findings from other studies on LBP in pregnancy by Emilia et al and Ferreira et al ${ }^{3,16}$ This observation explains LBP as an effect of alterations in the musculoskeletal system including postural changes, increasing load on the spine due to the growing fetus and the exaggerated lordosis that exert physical force over the spinal joints and causing dysfunction ${ }^{16,17}$. Maternal education level was associated with LBP such that those who attended secondary (adjusted OR 1.13, $p=.85$ ) and tertiary education (adjusted OR $1.30, p=.69$ ) had a $13 \%$ and $30 \%$ increased odds of developing LBP respectively, compared to those with no education at all. However, the results were not significantly different between the groups. Our results are similar to those reported by Wang et $\mathrm{al}^{4}$ and Emilia ${ }^{3}$ et al in the United States of America (USA) and Brazil, respectively. Women who had attended formal education were more likely to report LBP compared to their non-educated counterparts. A similar observation was reported among parturient women from Nepal and Nigeria where women with no formal education reported lowest pain scores compared to those with higher education ${ }^{18,19}$.

Low back pain affects negatively the ability of a pregnant woman to perform the activities of daily living such as self-care, walking, sitting and engagement in sexual activities. These functional limitations are also associated with reduced quality of life and reduced productivity among pregnant women. Our results are consistent with findings from Pakistan, the USA and Brazil where women also reported poor quality of life, limited daily functional activities and associated disablement due to pregnancy related LBP ${ }^{3,4,5}$. Similar results have also been reported by Gutke et al who reported that about $73 \%$ of pregnant women in Norway experienced mobility problems as a result of LBP and $\mathrm{PGP}^{20}$.

The current study also shows that there is limited clinical input in the management of LBP in pregnancy in Malawi. The majority of pregnant women resorted to self-management through the use of exercise, massage, stretching and self-prescribed analgesics. Our results agree with the findings of the study done in central Portugal where about $64 \%$ of pregnant women did not seek any treatment for their LBP because they did not know what help was available for them and they considered LBP as normal during pregnancy ${ }^{1}$. However, evidence-based management interventions for prevention and management of LBP in pregnancy exist. These interventions include manual therapy, stabilization, nerve stimulation and hydrotherapy often provided by physiotherapists ${ }^{5}$. Katonis et al argue that although it may not be possible to cure LBP completely, its burden on functional abilities could be adequately minized ${ }^{5}$.

\section{Implication of the study}

This study highlights the need to conduct routine clinical assessment for LBP among pregnant women attending antenatal clinics in Blantyre urban. Also, special focus needs to be given to pregnant women in second and third trimesters of pregnancy because of the reported higher odds of having LBP at these higher gestational ages. Furthermore, younger women need special attention as the findings of this study revealed increased odds of developing LBP than older women. Results from this study further highlight a need for thorough functional assessments during routine management of pregnant women ${ }^{5}$.

About $89 \%$ of the respondents in this study were not asked about their experience of LBP by the health care providers. Therefore, it is not surprising that the majority of pregnant women did not seek or get medical help for their LBP. Therefore, health care workers need to be proactive in asking about experiences of LBP and make a proper management plan which includes referral to appropriate health care workers to prevent and manage the LBP appropriately. Furthermore, health education and advice on LBP and its associated effects to pregnant women should be integrated as routine element of prenatal care. Proper referrals should also be made to specialist clinics for those with persistent symptoms ${ }^{1}$.

\section{Study limitations}

This was a facility based study and, as such, the prevalence reported may not be inferred to the general population of pregnant women because it only included those who attended antenatal clinics. Furthermore, this was a "self-report" prevalence study; it is likely results were affected by ascertainment bias which may compromise the true estimate of LBP in the study population. The LBP self-assessment used in this study may also affect the true LBP estimate within the population due to variations in interpretation of questions by respondents, since about $11 \%$ of the respondents had no formal education. The study also recruited participants from an urban setting only; therefore, results may not reflect the rural situation for pregnant women as they may be characteristically very different. Due to 
the nature of the study, results could be affected by reverse causation in that it was a cross-sectional study which may not explain the temporal relationship between LBP experience and pregnancy in this population.

\section{Conclusion and recommendations}

LBP is highly prevalent among pregnant women attending antenatal clinic in urban Blantyre, Malawi. Many pregnant women suffer considerable pain and discomfort during pregnancy. These experiences result into reduced social engagement, self-productivity and disablement. Given the prevalence and the clinical importance of LBP in pregnancy, health workers should be proactive in asking women about LBP experiences in order to provide necessary clinical care for their pain. Furthermore, health workers and researchers need to collaborate in their work towards improving women's health through research on prevention and treatment of LBP. Future studies should consider a wider variety of settings to incorporate the varied LBP experience in pregnant women and to elucidate the experience of living, seeking help and receiving treatment for LBP during pregnancy in Malawi.

\section{Conflict of interest}

Adamson Muula is the Editor-in-Chief of the Malawi Medical Journal, but was not involved in the peer review of this manuscript. The other authors declare no conflict of interest.

\section{Acknowledgement}

We would like to appreciate the women who voluntarily participated in this study, management of Queen Elizabeth Central Hospital and Blantyre District Health Office for granting us the permission to conduct the study within their premises, Ms. J Khaki who assisted with data analysis, the research assistants who helped with data collection, and the Africa Center of Excellence in Public Health \& Herbal Medicine (ACEPHEM) at the College of Medicine for the grant and technical support throughout the study implementation.

\section{References}

1. Mota M, Sá-couto P, Demain S. Women's experiences of low back pain during pregnancy. J Back Musculoskelet Rehabil. 2015;28(2):351-357. doi: 10.3233/BMR-140527.

2. Abebe E, Singh K, Adefires M, Abraha M. History of Low Back Pain during Previous Pregnancy Had an Effect on Development of Low Back Pain in Current Pregnancy attending antenatal care clinic of the University of Gondar Hospital, Northwest Ethiopia. Jour of Med Sc \& Tech. 2014;3(3). 37-44

3. Emília M, Costa C, Cavalcanti L, Alves C, Terceiro DL, Ravy D, et al. Low back pain during pregnancy. Rev Bras Anestesiol. 2017;67(3):266-270. doi.org/10.1016/j.bjane.2015.08.014

4. Wang S, Dezinno P, Maranets I, Berman MR, Caldwell-a drews AA, Kain ZN. Low Back Pain During Pregnancy :

Prevalence, Risk Factors, and Outcomes. Obstet G necol. 2004;104(1):65-70. doi: 10.1097/01

AOG.0000129403.54061.0e

5. Katonis P, Kampouroglou A, Aggelopoulos A, Kakavelakis K, Ly oudis S, Makrigiannakis A, et al. Pregnancy-related low back pain Hippokratia. 2011; 15(3):205-210

6. Norén L, Johansson G, Östgaard HC. Lumbar back and posterio pelvic pain during pregnancy : a 3-year follow-up. Eur Spine J. 2002: 11(3) :267-271. doi: 10.1007/s00586-001-0357-7
7. Gorginzadeh M, Imani F, Safari S. Pregnancy-Related Pelvic Pain: A Neglected Field in Developing Countries. Anesth Pain Med. 2016: 6(1): e35506. doi: 10.5812/aapm.35506

8. Ayanniyi O. Low Back Pain in Pregnancy: The Reality and the Challenge. Academic Journals. 2013;4(1):27-39.

9. Nesto T, Diener I. Patients with low back pain in Malawi : Their attitudes and beliefs on their low back pain. Clinical Medicine $\mathrm{R}$ search. 2014:3(4):112-118. doi: 10.11648/j.cmr.20140304.16 10. Khan MJ, Israr A, Basharat I, Shoukat A, Mushtaq N, Farooq H. Prevalence of Pregnancy Related Low Back Pain in Third Trimeste and Its Impact on Quality of Life and Physical Limitation. J Islam Int Med Coll. 2017;12(1):39-43.

11. Muula A. How do we define "rurality" in the teaching on medical demography? Rural Remote Health. 2007;7(1):653.

12. Ayanniyi O, Sanya AO, Ogunlade SO. Prevalence and Pattern of Back Pain among Pregnant Women Attending Ante-Natal Clinics in Selected Health Care Facilities. Afr. J. Biomed. Res. 2006: 9: 149-56 13. Yeoh PL, Hornetz K, Dahlui M. Antenatal Care Utilisation an Content between Low-Risk and High-Risk Pregnant Women. Plos: $2016(11) 3 ; 1-17$.

14. Vianin M. Psychometric properties and clinical usefulness of the Oswestry Disability Index. J Chiropr Med. 2008:161-3. doi org/10.1016/j.jcm.2008.07.001

15. Niskanen RO. The Oswestry low back pain disabil ty questionnaire: a two year follow up of spine surgery $\mathrm{p}$ tients. Scand J Surg. 2002;(5):208-11. doi

pdf $/ 10.1177 / 145749690209100214$

16. Ferreira W, Rodrigues G, Rangel L, Aparecida M, Nascime to DL, Soares C, et al. Prevalence of lower back pain and physical i activity : the impact of psychosocial factors in pregnant wome served by the Family Health Strategy. Einstein (Sao Paulo). 2011;9(4):489-93. doi: 10.1590/S1679-45082011AO2186 17. Nabih M, Gharib EL, Aglan AD. Changes in Skeletal Sy tem during Pregnancy. Int Gyn \& Women's Health 2(1)2018.IGWHC. MS.ID.000127. doi: 10.32474/I

WHC.2018.02.000127

18. Shrestha I, Pradhan N, Sharma J. Factors Influencing Perception of Labor Pain among Parturient Women at Tribhuvan University Teac ing Hospital. Nepal Journal of Obstetrics and Gynaecology. 2013;8(1). 26-30. https://doi.org/10.3126/njog.v8i1.8857

19. Olayemi O, Adeniji RA Udoh ES, Akinyemi OA, Aimakhu CO, Shoretire KA. Determinants of pain perception in labour among parturients at the University College Hospital, Ibadan. J Obstet Gynaecol. 2009: 25(2). 128-130. doi:

10.1080/01443610500040703

20. Gutke A, Boissonault J, Brook G, Stuge B. The Severity and Impact of Pelvic Girdle Pain and Low-Back Pain in Pregnancy : A Multinational. J Womens Health (Larchmt). 2018:27(4):510-517. doi: $10.1089 /$ jwh.2017.6342 